\title{
Car Accident Notification System based on Internet of Things
}

\author{
Priyal Raut \\ U.G. Student, Electronics and Telecommunication \\ Dept. Dwarkadas J. Sanghvi College of Engineering \\ Mumbai - 400056, India
}

\begin{abstract}
This paper proposes a distress call notification system using the Internet of Things and Cloud. It discusses the implementation of the proposed system using XBee WiFi module, Xbee Shield, GPS module, Seeeduino and crash sensors. The basic idea is to detect the occurrence of an accident using crash sensors, locate the exact position of the accident and communicate the co-ordinates of the location via Cloud using XBee WiFi to the nearest hospital.
\end{abstract}

\section{Keywords}

Internet of Things, Cloud, Seeduino, XBee WiFi, GPS, Crash Sensors, Server

\section{INTRODUCTION}

The Internet of Things is a global revolution. Introduced with the vision of uniting the physical world with the Internet, it has taken the world by a storm. With ubiquitous computing prevalent in every aspect of our lives, the IoT will add another link in this chain of global interconnectivity.We propose a system for distress call notification using cloud and SEEDUINO because this makes use of the mbed platform. The ARM mbed development platform is one which focuses on Internet of Things devices and was thus an ideal choice for our proposition. Our system is a proposition for Car Accident Notification of a single state.

The whole system makes use of a server (Cloud Computing) which allows the transition from simple, everyday monitoring systems to smarter, connected systems.

\section{BLOCK DIAGRAM}

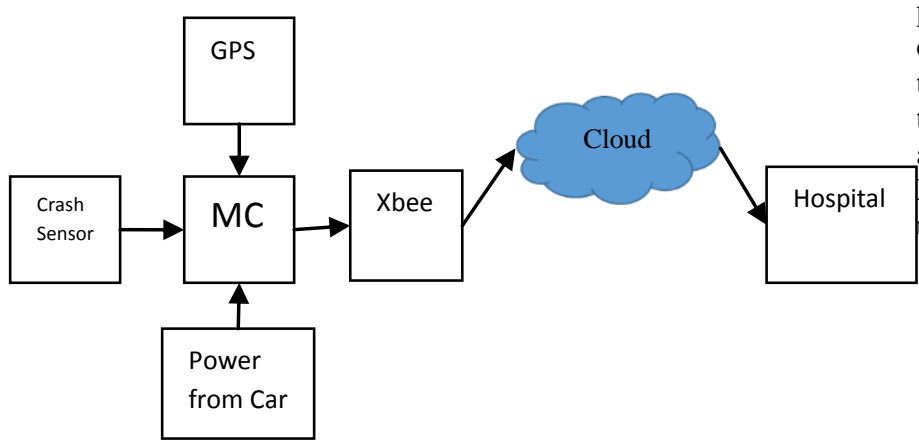

Figure 1: Block Diagram

\section{DESCRIPTION OF PARTS}

\subsection{Wireless XBee Shield}

An XBee Shield is used to enable the Seeeduino board to communicate wirelessly using XBee WiFi module. The XBee shield is connected to digital pins 0-7 and analog pins 0-5 on the Seeeduino development board. [4] It has a socket onto which the XBee WiFi module will be place. While uploading a sketch to the board with an XBee shield, the jumpers on the

\author{
Vanthana Sachdev \\ U.G. Student, Electronics and Telecommunication \\ Dept. Dwarkadas J. Sanghvi College of Engineering \\ Mumbai - 400056, India
}

shield need to be on the "USB" setting i.e. closer to the board edge.

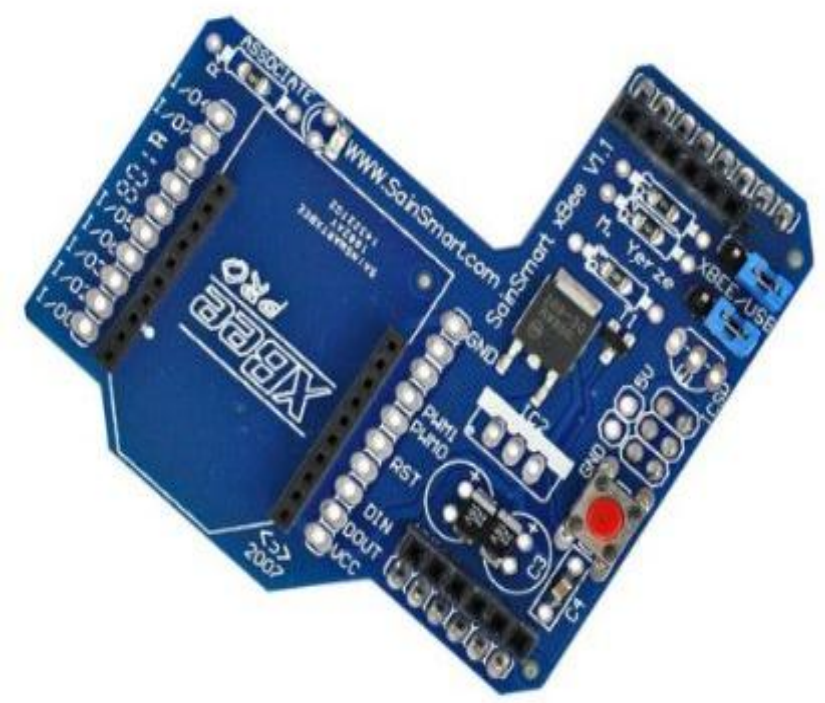

Figure 2: Wireless XBee Shield

\subsection{XBee WIFI Module}

The XBee WiFi embedded module has the ability to enable users to connect their devices to cloud using applications like Device Cloud by Etherios. [6] XBee WiFi has the same footprint as that of other XBee modules and can be used with the traditional XBee shields and thus offers flexibility. It provides serial to IEEE 802.11 connectivity. AT or API commands are used for configuration. XBee WiFi has overthe-air data rates upto $65 \mathrm{Mbps}$ and a wide operating temperature range from -40 to 85 degree Celsius. It has antenna options of RPSMA, PCB, U.FL and wire whip. The $\mathrm{XBee} \mathrm{WiFi}$ module is seated onto the appropriate socket on the XBee shield. 


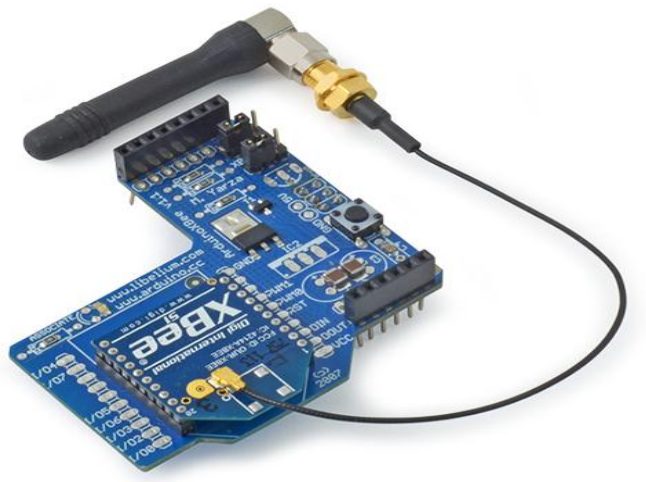

Figure 4: XBee Module with shield

\subsection{Seeeduino}

Seeeduino is an mbed enabled board which is completely compatible with the existing Arduino programs, shield and IDEs (Integrated Development Environment). The microcontroller used is ATMEGA168 and has a clock speed of $16 \mathrm{MHz}$. It has flash memory of $16 \mathrm{~KB}, \mathrm{SRAM}$ of $1 \mathrm{~KB}$ and EEPROM of 512 bytes. The seeeduino has 14 digital I/O pins and 8 Analog input pins. [2] The operating voltage is $5 \mathrm{~V} / 3.3 \mathrm{~V}$ and the recommended input voltage is $7-12 \mathrm{~V}$. Arduino environment is used to create and upload sketches on the development board. The two ways of power input are the USB cable and the external power jack. In case of greater memory requirement, Seeeduino Mega can be used. [3]

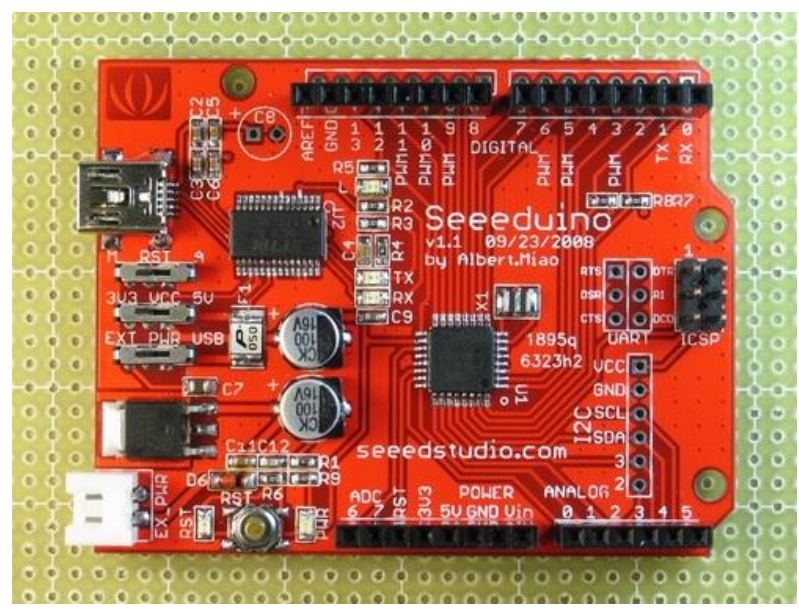

Figure 5: Seeeduino Board

\subsection{GPS Module}

The GPS module consists of the an electrical circuit which can be connected to an Arduino compatible board like Seeeduino and is used to get position, altitude as well as speed, date and time. It uses the standard NMEA protocol to transmit position via the serial port. The GPS module should be stored inside a plastic box and should be isolated from the environment. The antenna should be in horizontal position. Clear skies without any obstructions enable the GPS module to give accurate information. It is connected to the digital pins $8,9 . .13$, GND and AREF. It has to be powered separately as it does not receive power from these pins. [4]

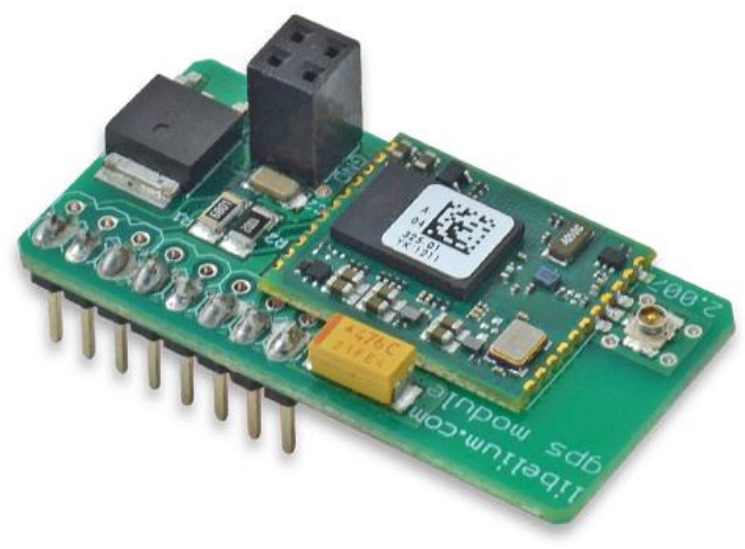

Figure 6: GPS Module

\section{WORKING}

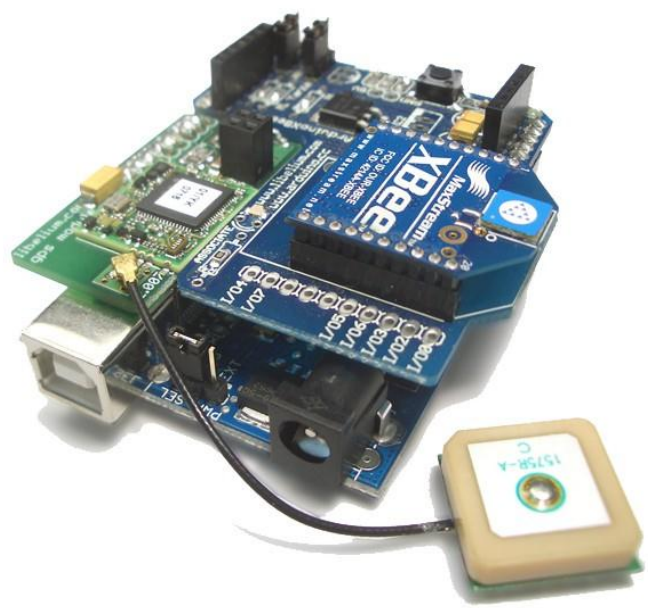

Figure 7: Entire system mounted on Seeeduino

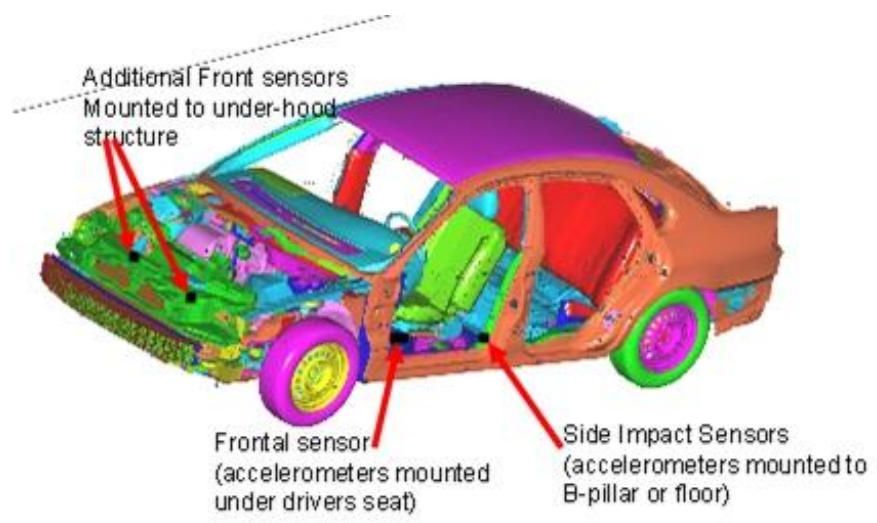

Figure 8: Placement of Crash Sensors

The crash sensors planted in the car are used to detect the occurrence of impact. If the impact is greater than a certain pre-defined threshold value, then the impact is considered to be an accident. The microcontroller ATMEGA168 is programmed to detect this accident. The GPS and the XBee modules are previously configured using the Arduino environment. On detecting the occurrence of the accident, the data obtained by the GPS is transmitted via XBee WiFi module. [9] 
This system requires a dedicated server which can be common to a whole state. The dedicated server will contain three data look up tables. One will have the car plate number and a space for location update. The second table will have the locations of all the hospitals in the state.

In the first table will be the Gateway Identifiers for all the XBee modules which will be able to communicate with the server. The XBee modules will be registered on the server on purchase of a car.

The hospitals will be able to $\log$ in to the server and register their locations in a look up table. This table will collectively hold the locations of all the hospitals of the state. For this

\section{LIMITATIONS}

a) The system requires a server for cloud computing which can be very costly, especially for large scale implementations.

b) The hospitals have to be logged into the server $24 \times 7$ and have to maintain a constant vigilance to the updates.

c) The cars need to be WiFi enabled.

\section{CONCLUSION}

Though the proposed system can be implemented using GSM technology, the idea was to propose a system allowing global interconnect with the Internet of Things and Cloud. [9] [10] .Despite the limitations mentioned above, the system is a step forward in the participation of realising the Internet of Things on a wider basis. With the use of cloud application, the data can be transmitted to longer distances. This application can be further improvised by programming the system to also notify the immediate family members of the accident victim.

\section{ACKNOWLEDGMENTS}

We would like to thank the respected principal Dr. Hari Vasudevan of D. J. Sanghvi College of Engineering and supporting us whilst we undertook the writing of this paper. We would also like to thank S.V.K.M. for encouraging us in such co-curricular activities.

\section{REFERENCES}

[1] XBee Wi-Fi Modules Available at http://www.digikey.com/product-highlights/us/en/digiinternational-xbee-wi-fi/108

[2] Seeduino V0.9bAvailable at http://ieee.rutgers.edu/system/files/Hello_Seeeduino.pdf

[3] Seeduino V2.21 Available at http://www.seeedstudio.com/depot/Seeeduino-V221Atmega-168P-p-690.html application to work, the hospitals will have to be logged into the server $24 \times 7$.

Once a module has been registered on the server, the server will continue to monitor information received by this module. If the signal of a car having met with a crash is received along with its location via the internet, the server automatically performs matching of the car location with the locations of the hospitals. It will be programmed to find the nearest match and immediately send a notification of the crash to that hospital. [9]

[4] How to use XBee and GPS with Arduino Uno Available at http://www.cooking-hacks.com/blog/tutorial-arduinodeluxe-pack

[5] XBee Module and Cloud Available at http://www.digi.com/xbee/

[6] XBee Wifi Embedded RF Modules Available at http://www.mouser.in/new/DigiInternational/digixbeeWIFI/

[7] [7] Luigi Atzori, Antonio Lera, Giacomo Morabito, 2010. Internet of Things: A Survey, Science Direct

[8] [8] Luis Ostiz Urdiain, Carlos Pita Romero, Jeroen Doggen, Tims Dams, Patrick Van Houtven, 2012. Wireless Sensor Network Protocol for Smart Parking Application Experimental Study on the Arduino Platform, Ambient 2012, The Second International Conference on Ambient Computing, Applications, Services and Technologies

[9] [9] Mr. Akshay Keshwatkar, Mr. Vishwa V, Mr.John Williams.R, Ms. P.S.Smitha, 2014. Sensor Based Automated Accident Tracking System, International Journal of Advanced Research in Computer Science Engineering and Information Technology

[10] [10] Ms. Kajal Nandaniya, Mr. Viraj Choksi, Dr. M. B. Potdar, 2014. Microcontroller Based Collision Detection and Warning System, International Journal of Advanced Research in Electrical, Electronics and Instrumentation Engineering

[11] Placement of Crash Sensors Diagram (Figure 8)Available at http://www.tasanet.com/knowledgeCenterDetails.aspx?d ocTypeID=1\&docCatID=41\&docID=197 